\title{
Calculation and design of tunnel ventilation systems using a two- scale modelling Approach
}

\author{
F. Colella ${ }^{a *}$ G. Rein ${ }^{b}$, R. Borchiellini ${ }^{a}$, R. Carvel ${ }^{b}$, J.L. Torero $^{b}$, V. Verda ${ }^{a}$ \\ ${ }^{a}$ Politecnico di Torino, Dipartimento di Energetica, Italy \\ ${ }^{\mathrm{b}}$ University of Edinburgh, BRE Centre for Fire Safety Engineering, UK
}

\begin{abstract}
This paper develops a novel modelling approach for ventilation flow in tunnels at ambient conditions (i.e. cold flow). The complexity of full CFD models of low in tunnels or the inaccuracies of simplistic assumptions are avoided by efficiently combining a simple, mono-dimensional approach to model tunnel regions where the flow is fully developed, with detailed CFD solutions where flow conditions require 3D resolution. This multiscale method has not previously been applied to tunnel flows. The low computational cost of this method is of great value when hundreds of possible ventilation scenarios need to be studied. The multi-scale approach is able to provide detailed local flow conditions, where required, with a significant reduction in the overall computational time. The coupling procedures and the numerical error induced by this new approach are studied and discussed. The paper describes a comparison between numerical results and experimental data recorded within a real tunnel underlining how the developed methodology can be used as a valid design tool for any tunnel ventilation system. This work sets the foundations for the coupling of fire-induced flows and ventilation systems where further complexities are introduced by the hot gas plume and smoke stratification.
\end{abstract}

\section{Introduction}

Tunnel safety is a concern in normal operating conditions as well as in emergency conditions (i.e. in case of fire). In both cases the ventilation system plays a fundamental role. In normal operating conditions it has to keep air quality acceptable and the concentration of pollutants within safety levels. In emergency conditions, its role is to control or extract smoke to allow fast and safe evacuation and safe fire fighting activities.

Different types of ventilations systems are commonly used in modern tunnels mainly depending on their characteristics, layouts and traffic flows [1,2]. Transverse and longitudinal ventilation systems are the most commonly adopted. Transverse ventilation systems use dedicated ducts for fresh air supply and extraction and they are well suited for long tunnels with bidirectional traffic. They have to be designed to assure smoke confinement in a small section of the tunnel keeping the air velocity low to enhance their stratification and provide effective extraction. Longitudinal ventilation systems use jet fans installed within the tunnel bore (generally on the tunnel ceiling) to provide longitudinal smoke control. They are highly effective when used in unidirectional traffic tunnels. In the case of longitudinally ventilated tunnels the air velocity has to be high enough to prevent smoke spreading against the flow direction. The phenomenon of smoke spreading against the prevailing flow is commonly known as back- layering. Prevention of back-layering can be achieved if the velocities generated by the system are higher than a threshold, known as the critical velocity [3-6].

Different methods can be used to design a ventilation system. Full scale tests, generally conducted within unused tunnels, require very large financial investments but provide large amounts of collected data. Some examples are the Memorial tunnel fire ventilation test program (MTFVTP) [7] and the EUREKA 499 firetun project [8]. Because of the huge costs associated, only a limited number of tests can be carried out. Design procedures sometimes use small scale tunnel models in order to represent ventilation and fire scenarios. Interpretation of their results is dependent on the relevant scaling laws and model scale results may not have a general validity in relation to the full scale case. The design of tunnel ventilation systems can be also conducted using numerical models. In this case, the designer has to deal with huge dimensions of the domain, intricate network of ventilation ducts, the presence of any kind of obstacles or blockages and changeable boundary conditions (wind at the adits and variable static pressure). Furthermore, all the possible settings of all the ventilation devices, fans, dampers, etc. mean that the number of ventilation scenarios can be very large. Thus, the design of tunnel ventilation systems is still a challenging issue. Given all the modelling complexities, the first step is generally to model steady state flows within a tunnel, under ambient conditions, and then move to more complex 


\begin{tabular}{|ll|}
\hline \multicolumn{2}{|l|}{ Nomenclature } \\
$a, b, c$ & fan characteristic curve coefficients \\
$D_{h}$ & branch hydraulic diameter [m] \\
$f$ & major losses coefficient \\
$G$ & mass flow rate through a branch $[\mathrm{kg} / \mathrm{s}]$ \\
$G_{\mathrm{cfd}}$ & mass flow rate computed using the full CFD \\
& approach $[\mathrm{kg} / \mathrm{s}]$ \\
$G_{\text {ext }}$ & mass flow rate exchanged in a node $[\mathrm{kg} / \mathrm{s}]$ \\
$G_{\mathrm{ms}}$ & mass flow rate computed using the Multi-scale \\
& model $[\mathrm{kg} / \mathrm{s}]$ \\
$g$ & gravity acceleration [m/ $\left./ \mathrm{s}^{2}\right]$ \\
$L$ & branch length [m] \\
$p$ & pressure [Pa] \\
$v$ & velocity [m/s] \\
$z$ & node elevation [m] \\
$b$ & minor losses coefficient \\
$e_{\mathrm{ms}}$ & Error induced by the Multi-scale model [\%] \\
$\bar{\rho}$ & branch average density $\left[\mathrm{kg} / \mathrm{m}^{3}\right]$ \\
$D p_{\mathrm{fan}}$ & pressure gain induced by jet fans [Pa] \\
$D p_{\text {loss }}$ & pressure losses [Pa] \\
\hline
\end{tabular}

scenarios involving fire, obstructions due too large vehicles, ventilation failure or fan operation delay times.

Different modelling approaches can be used. If details of the flow field are needed, $2 \mathrm{D}$ or $3 \mathrm{D}$ computational fluid dynamics (CFD) tools can be used to provide data of the flow behaviour around walls, ventilation devices and obstructions in the tunnel. The computational cost of this class of methods is high even for medium size tunnels and they are often only used for design verification. If the analysis requires only bulk flow velocities, monodimensional models can be used. This class of methods has been extensively used for design purposes. Their low computational cost favours large studies of all possible ventilation scenarios.

Another class of methods, called multi-scale methods, adopts different levels of complexity in the numerical representation of the system. This method has not been used before for tunnel flows. Regions of interest can be described using more detailed models, while the rest of the system can be represented using a simpler approach. In this work a mono-dimensional model and a CFD model have been combined together in an effective way and used to address some of the typical modelling problems encountered when dealing with tunnel ventilation flows.

\section{Modelling techniques for tunnel ventilation}

Many papers have been written in the last couple of decades about the application of monodimensional models to the design of tunnel ventilation systems. These methods use a simplified representation of the tunnel and ventilation ducts under the assumption that all the fluid properties are constant within each cross section. Mono-dimensional models give a poor representation when they have to deal with complex 3D flows generated by ventilation devices (i.e. jet fans) or by fire. On the other hand, given their low computational complexity, they can be adopted for parametric studies of the whole system including tunnel, chimneys, supply and extraction ducts, etc. In order to describe a tunnel layout, the common practice is to split the domain into branches and nodes linked together in a network.

Ferro et al. [9] presented a computer model applied to the fluid-dynamic simulation of a longitudinally ventilated tunnel. The same theoretical approach has also been used in the contribution by Jacques [10] presenting a numerical simulation of complex road tunnels. More recent applications of this methodology are presented by Riess et al. [11], Cheng et al. [12] and by Jang and Chen [13]. This last work [13] provides a methodology to estimate the aerodynamic coefficients of a road tunnel using both numerical and experimental data. All the previous contributions underline the strength of mono-dimensional model to investigate ventilation scenarios within tunnels.

On the other hand, just a limited number of field models applied to tunnels are described in the literature. Armstrong [14], performed a comparison between CFD results and experimental data provided by a small scale model. Tabarra [15] studied the flow generated by fans within a rectangular tunnel using CFD tools and compared the results with experimental data. Both of these works had to deal with isothermal flow and presented a good agreement between experimental and numerical data. Karki and Patankar [16] described an application of field models to simulate ventilation flows within a tunnel in a fire scenario. The model has been validated using data from the MTFVTP [7] and shows a good agreement with the measurements. The MTFVTP data [7] has also been used by Vega [17] to validate their CFD model which included ventilation devices.

The most important aspect of the CFD modelling of tunnel ventilation flows, is that to adequately simulate the fluid behaviour in a small portion of a domain, the flow conditions at locations far away from the region of interest are also required. In other words, the particular flow field that is established in a small region of the tunnel results from a complex coupling between ambient boundary conditions, ventilation devices and the whole tunnel structure. As a consequence of this, even if only a limited region of the fluid flow has to be investigated, the numerical model needs to take into account the whole tunnel domain and all the operating ventilation devices. In the case of a long tunnel this may mean that the computational domain has to include locations many kilometres away from the region of interest, resulting in a computational mesh including many millions of nodes. 


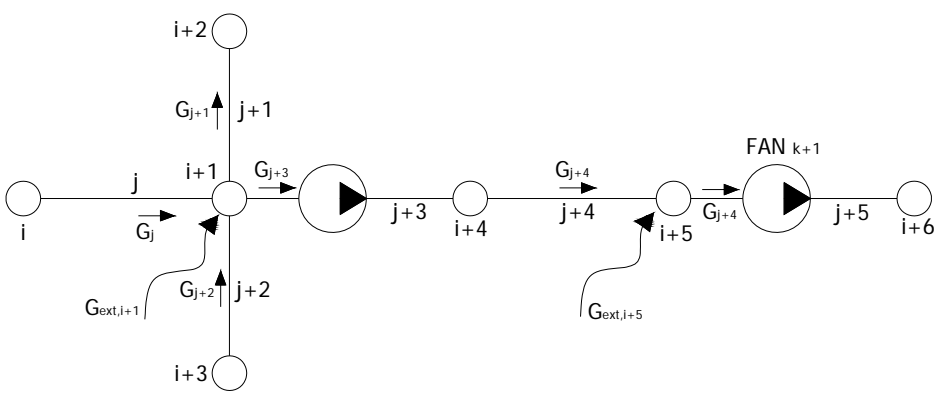

Figure 1 - Example of the network representation of a tunnel showing branches between nodes

The basic idea under the multi-scale methods is to consider the tunnel as a system where the fluid is behaving as fully developed as any flow through a pipe. The problem of a fully developed flow in a duct has a simple closed form solution, has been studied before and can be easily solved using mono-dimensional models. Non fully developed flows are encountered in some regions of the tunnel, for example near the portals, a shaft or downstream of the jet fans, and are formed due to large disturbances in the flow. The flow here is not mono-dimensional but is $2 D$ or $3 D$ in nature. These are the regions where more powerful tools such as CFD techniques are required. This approach allows a significant reduction of the computational time because the more time consuming tools are not applied to the entire domain, but only to the regions where they are needed. The rest of the domain is modelled using a mono-dimensional tool. No contributions are known where multi-scale modelling techniques have been adopted to simulate ventilation flows within a tunnel. However, this modelling approach has been extensively used for simulations of blood flow in the circulatory system (e.g. Formaggia et al. [18,19]).

\subsection{Monodimensional model}

The first step is to define a network of elements representing the tunnel layout whose domain has been discretised in oriented elements called branches, interconnected by nodes.

The nodes are volume-less elements connecting different branches. Each node is characterized by vertical elevation and by the value of mass flow rate exchanged with the ambient (leaving or entering the domain). Pressures, densities and temperatures are defined in each node.

The branches are oriented elements connecting two nodes. The characteristics of each branch are section, perimeter, length, wall roughness, a coefficient for minor losses and pressure gain due to fan action. Velocities and mass flow rates are defined for each branch. The governing relations required to solve the fluid-dynamic problem are the mass and momentum conservation equations, expressed for steady state and incompressible flow. The developed model solves the mass conservation equations at each node and the momentum conservation equation across each branch.

An example of a general network layout is presented in Fig. 1 where nodes are numbered as $i$ and branches as $j$.

The mass conservation equation states that for each node the mass flow rate added or subtracted by connecting branches has to be equal to the mass flow rate exchanged in the node with the external ambient

$\sum_{j} G_{j}=G_{e x t, i}$

where the summation is extended to all the branches connected to the node $i$.

The momentum conservation equations are written for each branch following the well known Bernoulli formulation. Given a generic branch $j$, included between nodes $i$ and $i+1$, the longitudinal momentum equation states

$$
\begin{aligned}
p_{i+1}-p_{i} & =\Delta p_{j_{\text {loss }, j}} \\
& =-\Delta p_{f a n, j}-\bar{\rho}_{j}\left(\frac{v_{i+1}^{2}-v_{i}^{2}}{2}\right)-\bar{\rho}_{j} g\left(z_{i+1}-z_{i}\right)-\Delta p
\end{aligned}
$$

The pressure gain induced by the fans and the pressure losses induced by the wall friction (major losses) and blockages (minor losses) need a mathematical description in order to correlate them to the air velocity through the specific branch. Following $[9,10,13]$, the pressure losses can be expressed as

$\Delta p_{\text {loss }, j}=\overline{\rho_{j}} \cdot \frac{v_{j}^{2}}{2}\left(\frac{L_{j}}{D_{h, j}} f_{j}+\beta_{j}\right)$

where the friction coefficient is computed using the Colebrook formula [20].

The pressure gain induced by the fans is a nonlinear function of the velocity within the branch and can be represented as a polynomial of second or third order $[9,10,13]$ as 


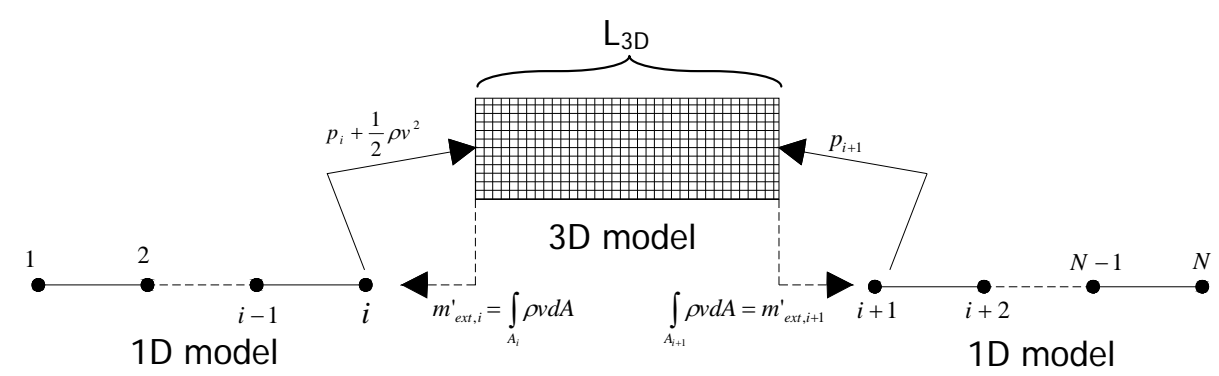

Figure 2 - Boundary conditions for the direct coupling of the 1D and CFD models

$\Delta p_{f a n, j}=a+b \cdot v_{j}+c \cdot v_{j}^{2}$

In most of the cases the fan characteristic curves are completely unknown or not well defined because their behaviour is strongly dependent on their surroundings and their installation [13]; distance from the ceiling, eccentricity, etc. Usually, in situ measurements or further CFD analysis are required to adequately define their behaviour.

\subsection{CFD model}

In this work all the CFD results are obtained using the finite volume CFD tool FLUENT [21]. In this particular application a segregated solver has been used to solve the Navier-Stokes equations. The fluid has been considered as incompressible and the SIMPLE method presented by Patankar and Spalding [22] has been adopted to solve the link between pressure and velocity fields.

In order to take care of the complex turbulent phenomena within the domain, two more additional equations for the turbulent quantities need to be solved. The $k-\varepsilon$ turbulence model [23] has been largely validated by the scientific community and it is still the most practical and least time consuming approach.

The standard $k-\varepsilon$ model is not valid for fluid regions characterized by a low Reynolds number, like locations close to the walls [24] (in these regions the standard wall functions have been used). This approach provides adequate results for flows with high Reynolds numbers [25,26] avoiding the need to use a very high resolution mesh to solve the viscous sub-layer by making use of the universal behaviour of the near wall flows (Schlichting [26]). The wall functions approach is valid as long as the first mesh point is located within the logarithmic region of the boundary layer. This constraint has to be considered when a near wall mesh has to be generated. A posteriori computations have been performed in order to check that the dimensionless distance of the first mesh point $y^{+}$ranges between 30 and $300[25,26]$.

The evaluation of the fluid-dynamic quantities at the finite volume interface uses a second order upwind scheme for all the convective terms and for the pressure [21].

The CFD model also requires a representation of the jet fans. Previous works on the subject $[16,17]$ simulated the jet fans as a combination of sources and sinks. This kind of approach has not been used in this paper in order to avoid discrepancies in energy, mass and momentum that can be generated by uncoupling discharge and intake surfaces. The proposed methodology simulates the jet fans as fluid regions delimitated by cylindrical walls and containing a cross-sectional surface where a constant, positive pressure jump is imposed.

The three dimensional tunnel domain has been discretized using a quasi-structured mesh with some refinements introduced in the proximity of the jet fans. The solution has been assumed to be converged when the scaled residuals were lower than $10^{-5}$ with the exception of the energy equation, in which case the maximum allowed value was $10^{-7}$.

\subsection{Multi-scale model}

The multi-scale model is generated by coupling a mono-dimensional Network model with a CFD model. The great advantage of this class of methods is that they produce a significant reduction of the computational time due to a more rational use of the computational resources. Regions of the domain where the flow is behaving as fully developed (far field) are modelled by using a mono-dimensional approach while more computational resources are dedicated to solve the regions where the flow has a complex $2 \mathrm{D}$ or 3D pattern (near field).

The coupling between mono-dimensional and CFD models can be obtained in two different ways; direct and indirect coupling.

Direct coupling is required if details of the flow field must be calculated. In this case the monodimensional model has to be embedded within the CFD model. The solution of the multi-scale model requires a constant exchange of information between the 1D and the 3D models at the interfaces, during the solving procedure (Fig. 2). The coupling algorithm, operating at the interfaces between the 1D and 3D models at nodes $i$ and 
$i+1$, has to perform the following operations at each iteration step:

1. provide the CFD model with pressure boundary conditions at the interfaces $i$ and $i+1$ calculated by the monodimensional model

2. perform one iteration of the CFD model using a segregate solver

3. integrate the air velocities at the interfaces $i$ and $i+1$ to calculate the global mass flow rate and fix them as boundary for the mono-dimensional model

4. solve the mono-dimensional model

5. repeat back to step 1 until convergence is reached.

6. proceed to the following time step (for transient calculations)

A more detailed overview of the sequence of operation required by the multi-scale model is presented in Fig. 3.

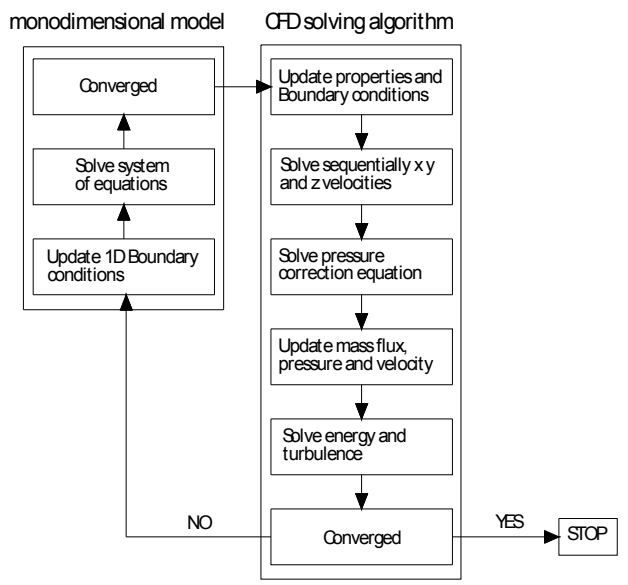

Figure 3 - Coupled solving algorithm

Any CFD model requires values for the turbulent kinetic energy and for the dissipation rate at the domain boundaries. These quantities are not calculated by the mono-dimensional model, so the coupling algorithm must take this into consideration. These are calculated as a function of turbulence intensities, turbulent length scale and Reynolds number using well known relations that can be found in the literature [24].

The 3D regions must be sized appropriately, such that the boundary interfaces are located in regions where the disturbances have disappeared, or are negligible, and the flow largely behaves in a mono-dimensional fashion.

At the boundary interfaces, therefore, instead of fully developed profiles of the fluid-dynamic quantities, flat profiles may be used. Nevertheless, if the interfaces are far from region of interest, there is no appreciable effect on the final solution because the flow has sufficient longitudinal distance to attain a fully developed profile. However, there is generally a small effect induced by the interface location; this has been calculated for the test case and will be presented in the results section.

In this work, a multi-scale model with direct coupling has been applied to calculate the flow field produced by an operating pair of jet fans in a real tunnel. The numerical results provided by the model are compared to experimental data.

Most of the comprehensive ventilation studies require only bulk flow velocities or temperature within the tunnel. In this case an indirect coupling method can be adopted and mono-dimensional and CFD simulations can be run separately. First, the near field to be modelled using CFD, must be identified. This usually coincides with the tunnel regions where the flow is behaving in a complex manner, and a simple mono-dimensional representation would lead to inaccurate results. After identifying the near field, many CFD runs are performed under a range of different boundary conditions. Generally, pressure boundary conditions must be imposed on the CFD model. After convergence is achieved, the CFD model is able to provide the bulk flow velocity through the domain. Also, in this case, it must be noticed that the boundary of the near fields must be placed in the fluid region where the flow is behaving in a monodimensional fashion.

Thus, the CFD results can be used to describe the flow behaviour of the near field as a function of the same average quantities required by the mono-dimensional model. Generally, for cold flows within a tunnel, the CFD data can be arranged in terms of bulk velocities and pressure differences across the near field allowing the definition of characteristic curves for the section of tunnel modelled using CFD. If the monodimensional model is able to take into account those curves, it is able to represent the fluiddynamic behaviour of the tunnel, exploiting the additional information provided by the CFD model and, hence, improving its modelling capabilities. Generally, direct coupling leads to longer computational times since the solving speed is set by the CFD model. Indirect coupling leads to higher set-up times but then almost real time calculations can be carried out once a set of characteristic curves have been calculated.

In this work a multi-scale model with indirect coupling has been applied to perform an analysis of a longitudinal ventilation system installed in a real tunnel. Also, in this case, the numerical results are compared to experimental data recorded in the tunnel.

\section{Application of multiscale methods to a $1.5 \mathrm{~km}$ long tunnel}




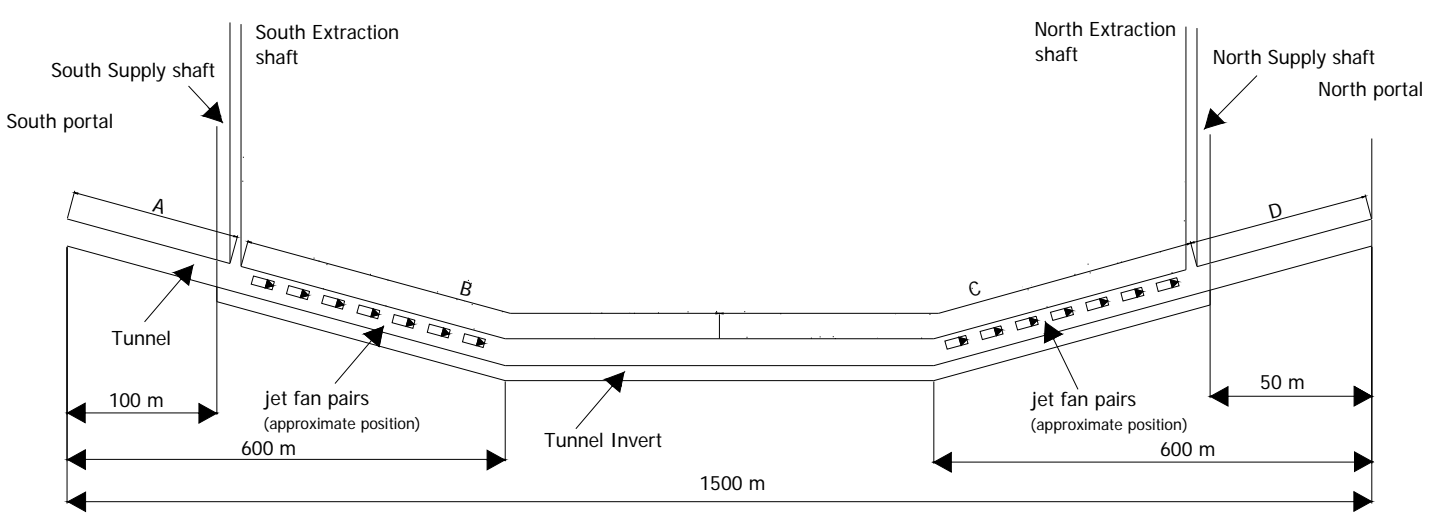

Figure 4 - Longitudinal layout of the tunnel showing the main elements of the ventilation system._Vertical dimensions not to scale.

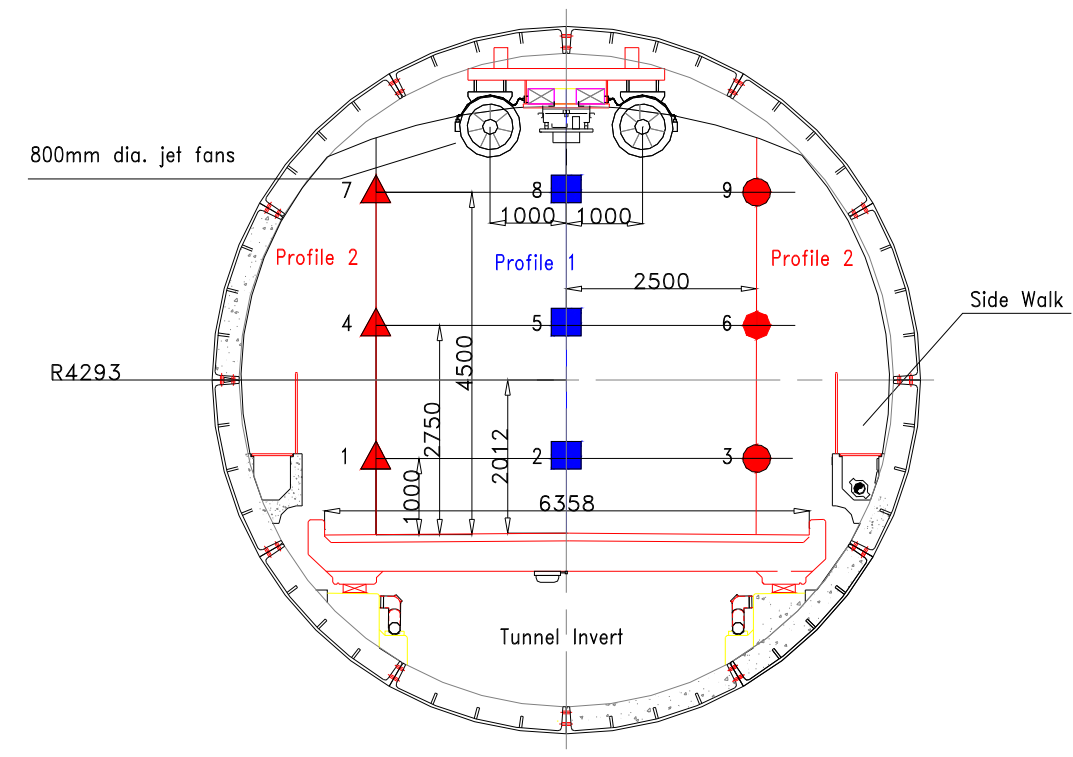

Figure 5 - Layout and general dimensions of the tunnel cross section including the points 1-9 where the air velocities were measured (dimensions are in $\mathrm{mm}$ ).

The tunnel considered is approximately $1.5 \mathrm{~km}$ long and carries unidirectional traffic in two lanes from the north portal to the south portal. The central portion of the tunnel is flat while the initial and the final segments present a descending and ascending layout. The cross section is around 40 $\mathrm{m}^{2}$ and it is slightly variable along the tunnel depending on the longitudinal position.

The tunnel is equipped with a semi-transverse ventilation system. Two shafts with axial extraction are located at a relatively short distance from each of the tunnel portals. The fresh air is pumped into the invert under the roadway by means of two axial fans located close to the adits. The supply grills are located along the side of the roadway between the two extraction shafts. The tunnel is also equipped with 14 pairs of jet fans. They are installed in two groups of 7 pairs in the inclined sections of the tunnel. The fan pairs are spaced around $50 \mathrm{~m}$ from each other and provide a volumetric flow rate of $8.9 \mathrm{~m}^{3} / \mathrm{s}$ with a discharge flow velocity of $34 \mathrm{~m} / \mathrm{s}$. Each fan pair is installed in a niche in the tunnel roof. Schemes of the tunnel longitudinal and transversal sections are presented in Figs. 4 and 5.

\subsection{Calculation of the jet fans near field}

An assessment of the performance of the tunnel ventilation system was undertaken requiring a detailed study of the flow field around the jet fans. In particular, an analysis of the jet fans discharge cone was required. These details are fundamental to understand how the jet fans interact with their surroundings and how installation (e.g. in niches) affects the final performance of the system and their capabilities of producing thrust. For this purpose an experimental campaign was 


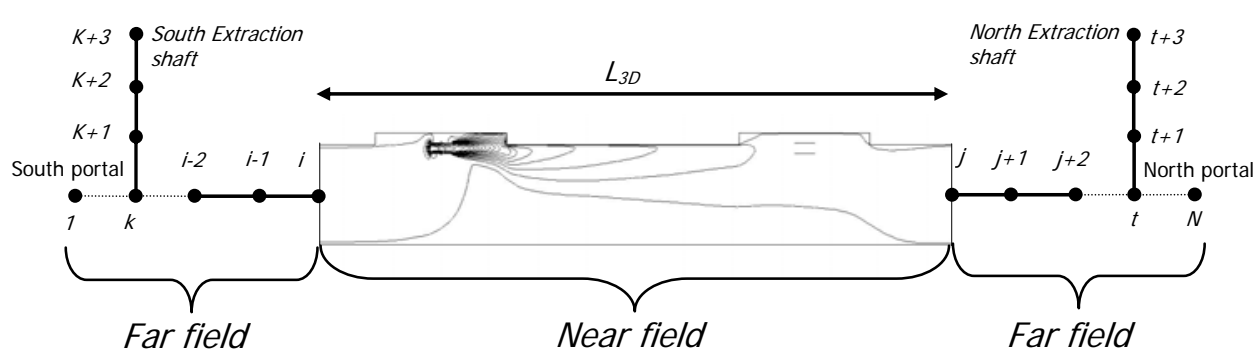

Figure 6 - Multiscale coupling between mono-dimensional and CFD models

performed and some modelling attempts were made in order to reproduce the flow field around the jet fans.

A multi-scale model has been applied to calculate the flow field generated by just one operating jet fan pair. As local details of the flow field are required direct coupling was used. For this analysis, the tunnel domain was split into a near field region where a CFD model was used and a far field region modelled using a monodimensional approach. A visual example of the model arrangement for this specific application is presented in Fig. 6. Neither the air supply network nor the other (non operational) jet fans were included in the model, in order to keep the analysis simple.

\subsubsection{Effect of the interface locations}

A sensitivity analysis has been conducted in order to asses how the position of the interfaces between mono-dimensional and CFD domain affects the calculated solution. An operating jet fan produces a region where the fluid field has high velocity gradients and a proper modelling approach would require a CFD tool. The high gradient region does not extend for a long distance and after a while the flow behaves as fully developed and it could be successfully represented using a mono-dimensional model. In this region the interface between monodimensional and CFD domain has to be located. In order to identify this distance, 14 different runs of the multi-scale model were performed. In each run the interfaces were placed progressively further away from the operating fans, increasing the longitudinal extension of the CFD domain $\left(L_{3 D}\right.$ Fig. 6) and consequently reducing the extension of the mono-dimensional domain. For each run the predict bulk flow (Fig. 7) has been recorded. The reference value is the bulk flow calculated using a full scale CFD simulation of the whole tunnel.

Defining L3D as the length of the near field, the error induced by an inappropriate location of the interfaces can be calculated as

$\varepsilon_{m s}=100 \cdot \frac{\left|G_{c f d}-G_{m s}\right|}{G_{c f d}}$ where $G_{c f d}$ is the mass flow rate calculated using the full CFD model, and $G_{m s}$ is the mass flow rate computed by the multi-scale model for different values of $L_{3 D}$. Fig. 7 shows the error calculated in each run and its dependence on the $3 D$ domain length. It is clear that the multiscale approach can lead to accurate results when the dimensions of the CFD domain are only a fraction of the whole tunnel length $(1.5 \mathrm{~km})$ with a significant reduction of the computational time. Results with less than $10 \%$ error can be obtained using a 3D domain longer than $80 \mathrm{~m}$ ( $5 \%$ of the tunnel length). The accuracy of the multi-scale model is improved up to around $1 \%$ by using 300 mas length of the near field (20\%of the tunnel length). For all the following calculations the length of the $3 \mathrm{D}$ model has been set to $300 \mathrm{~m}$.

\subsubsection{Effect of mesh size}

Various CFD runs have been also conducted to asses the mesh requirements. Four different meshes have been generated and the resulting solutions were compared. The mesh density per meter of tunnel length ranged from 272 cells $/ \mathrm{m}$ up to 7000 cells $/ \mathrm{m}$. The symmetry of the domain across the longitudinal plane was also considered. Four examples of the mesh cross section are presented in Fig. 8.

The calculated solutions have been checked at two positions within the computational domain located $10 \mathrm{~m}$ upstream and downstream of the operating jet fan pair, on the symmetry plane. Horizontal velocity predictions, plotted as function of the elevation from the road deck, are presented in Fig. 9. As can be seen, the calculated velocity pattern is not significantly different when calculated using meshes 3 and 4 , thus it is assumed that grid independence has been reached. Thus, all the simulations have been conducted using mesh 3 whose density was around 4000 cells $/ \mathrm{m}$.

\subsubsection{Comparison to experimental data}

The multi-scale model results were compared to experimental velocities measured using a rotating vane anemometer during an experimental campaign conducted in the tunnel. The horizontal 


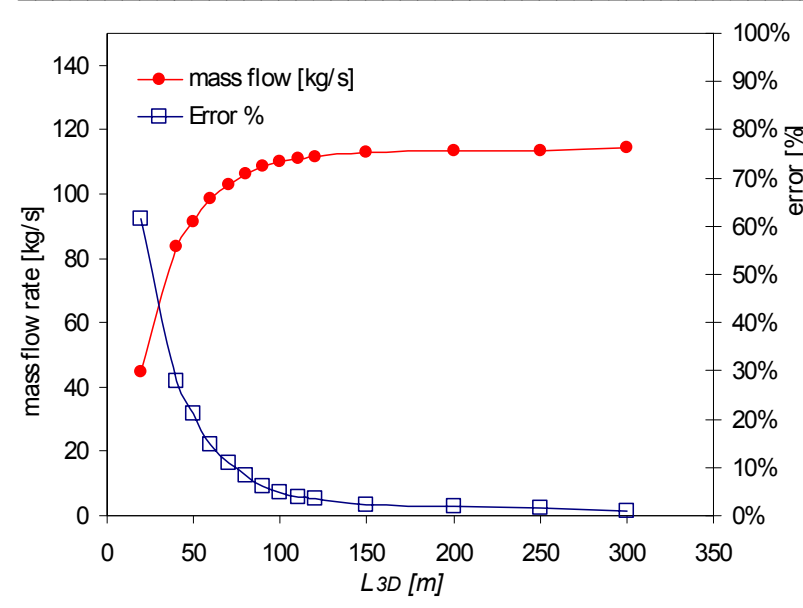

Figure 7 - Convergence of the predicted mass flow rate as a function of the location of the interfaces
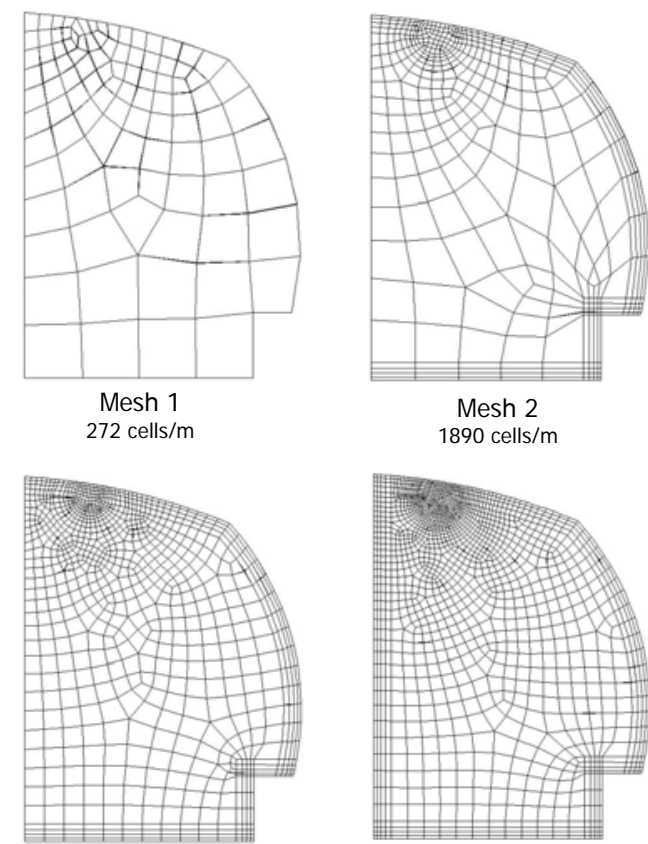

Mesh 3

4000 cells $/ \mathrm{m}$

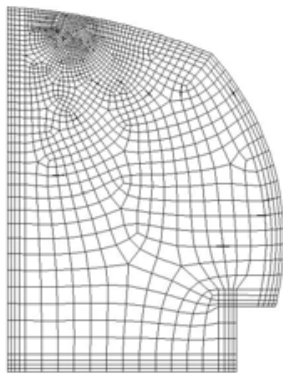

Mesh 4

7000 cells/m

Figure 8 - Examples of the different meshes used for half of the tunnel cross section and number of cells per unit length of tunnel.

air velocities have been recorded at various points across the cross section, at six different locations, at $20 \mathrm{~m}$ intervals, starting $20 \mathrm{~m}$ downstream from the jet fan discharge surface. In each section 9 velocity points have been considered. Fig. 5 shows the spatial location of the points where the velocities were measured.

The comparison between predicted and recorded velocities is presented in Fig. 10. The predicted values are represented by blue continuous and red dashed lines. The blue continuous line represents the velocity profiles calculated in the middle of the
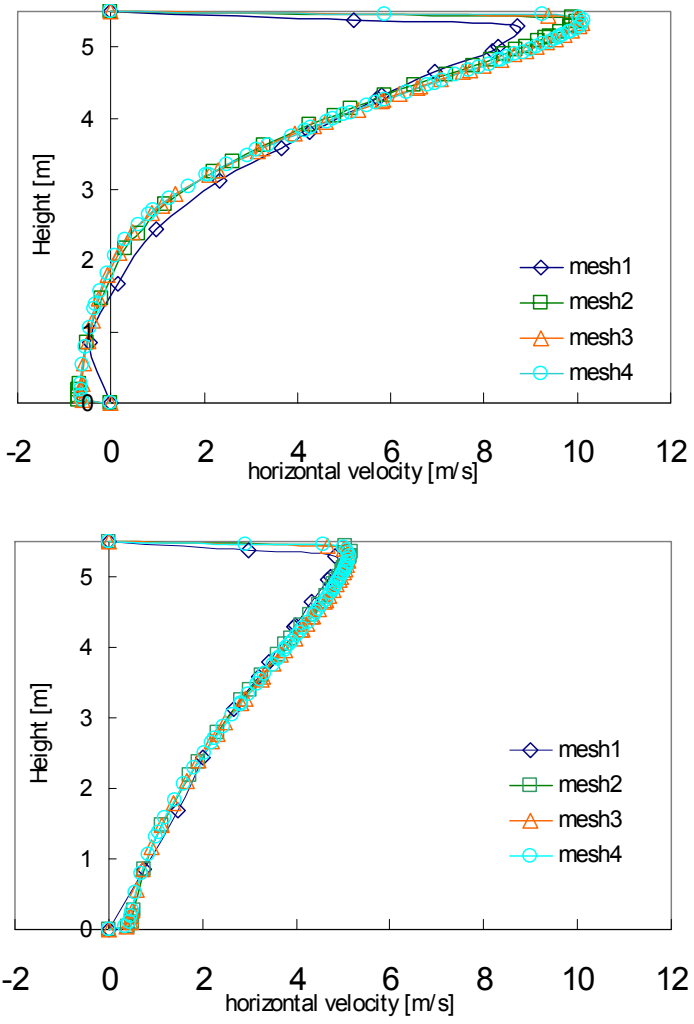

Figure 9 - Predicted profiles of velocity vs. height for different meshes; top $10 \mathrm{~m}$ downstream from the jet fan; and bottom $10 \mathrm{~m}$ upstream the jet fan.

tunnel cross sections (profile 1 in Fig. 5) while the red dashed ones represent the velocity profiles calculated along the vertical lines corresponding to the profiles 2 in Fig. 5. The measured velocity values represented in Fig. 10, are numbered from 1 to 9 following the same pattern as presented in Fig. 5. The comparison is quite encouraging as in almost all the measurement sections there is a good agreement between experimental and numerical data.

All the CFD tests done during the development of the model have demonstrated that the niches where the jet fans are located have a significant effect on the longitudinal development of the flow and their capability of producing thrust. The worst match has been recorded in section $60 \mathrm{~m}$ downstream from the fan pair; this peculiarity is most likely due to the presence of obstacles located on the tunnel ceiling (other fans and lighting devices) which are not included in the computational domain but will influence the discharge cone characteristics.

\subsection{Calculation of the performance of the ventilation system}

The assessment of the ventilation system performance required a comprehensive study of the ventilation strategies within the tunnel. In particular, the study aims to understand the consequences on the tunnel flow of making 

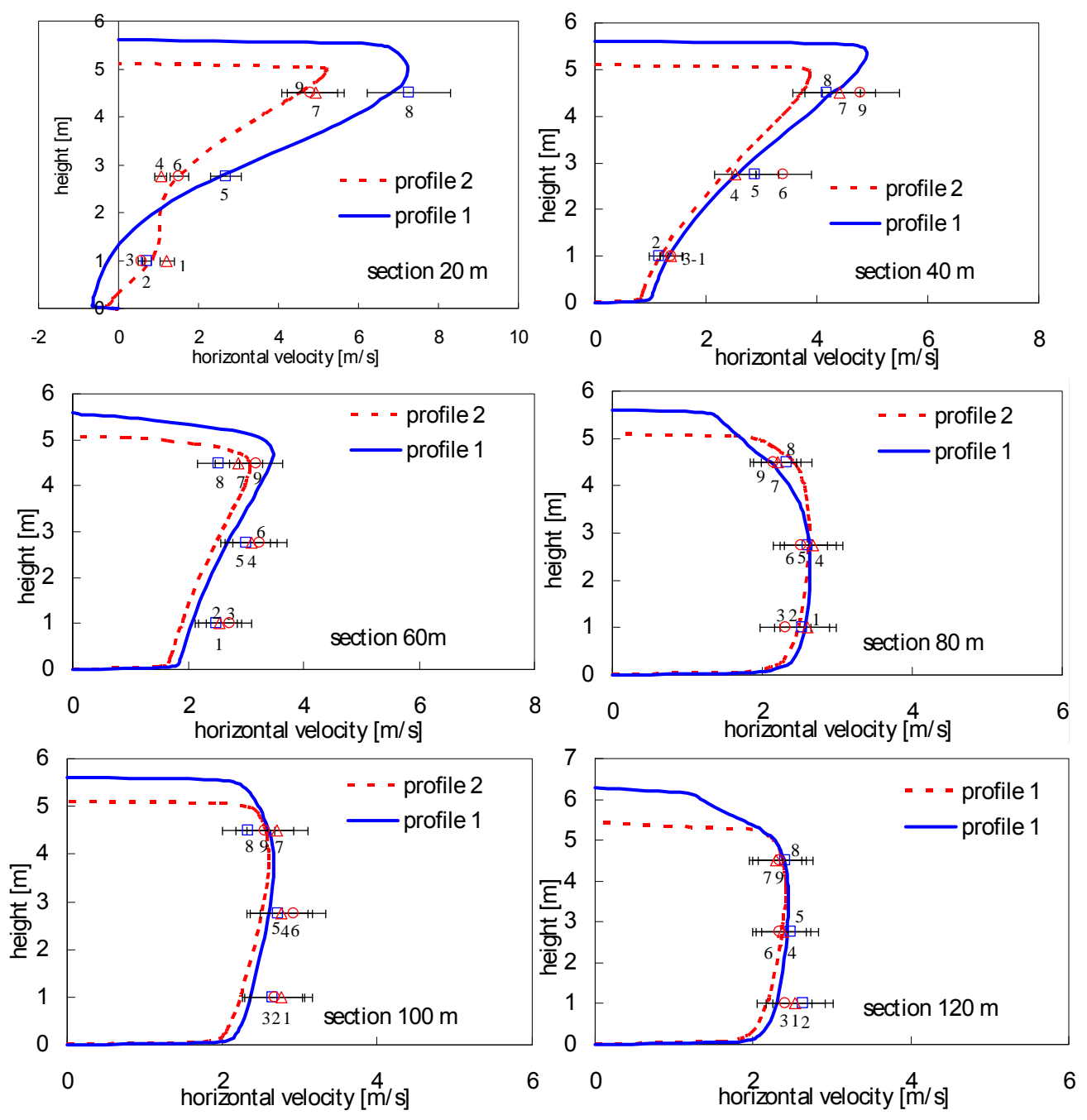

Figure 10 - Comparison of horizontal velocities between predictions (lines) and experimental measurements (symbols). The two profiles and the numbers refer to locations in the tunnel section described in Figure 5.

changes to the fan configurations. For this purpose, several simulations must be performed but only bulk flow velocities within the tunnel are required.

The first modelling choice to address this problem was a purely mono-dimensional model. For this particular application, the main difficulty encountered when using the mono-dimensional approach was related to the assessment of the jet fans thrust and their losses induced by their peculiar installation locations (i.e. in niches). In fact, it is well known that the pressure rise produced by the jet fans is strictly dependent on the surrounding environment [13]. Some empirical correlations to estimate the thrust from jet fan pairs were adopted $[9,10,12]$ but, in all the cases they over-predict the actual capabilities of the ventilation system.

In order to overcome this problem, a multi-scale modelling approach with indirect coupling was used. The region of high gradients, in this case, is represented by the fluid domain close to the operating jet fan pair. In case of the activation of many jet fan pairs, the flow within the tunnel domain is characterized by many regions of high gradients requiring to be modelled using a CFD approach. Obviously, depending on the number and location of the operating jet fans, different meshes for the near field must be built. To avoid this computational complexity, some preliminary CFD runs have been performed in order to understand how a series of active jet fan pairs operates. All the results have shown that a series of equidistant jet fans within a tunnel produces a flow field characterized by an almost periodic pattern. Fig. 11 is a clear example: velocity isocontours calculated for a series of 7 operating jet fans are presented and the periodicity of the flow field is evident.

As the flow periodicity has been accessed, the computational domain of the near field has been limited to the periodic portion of the tunnel geometry where 


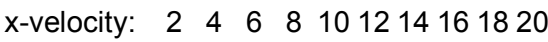

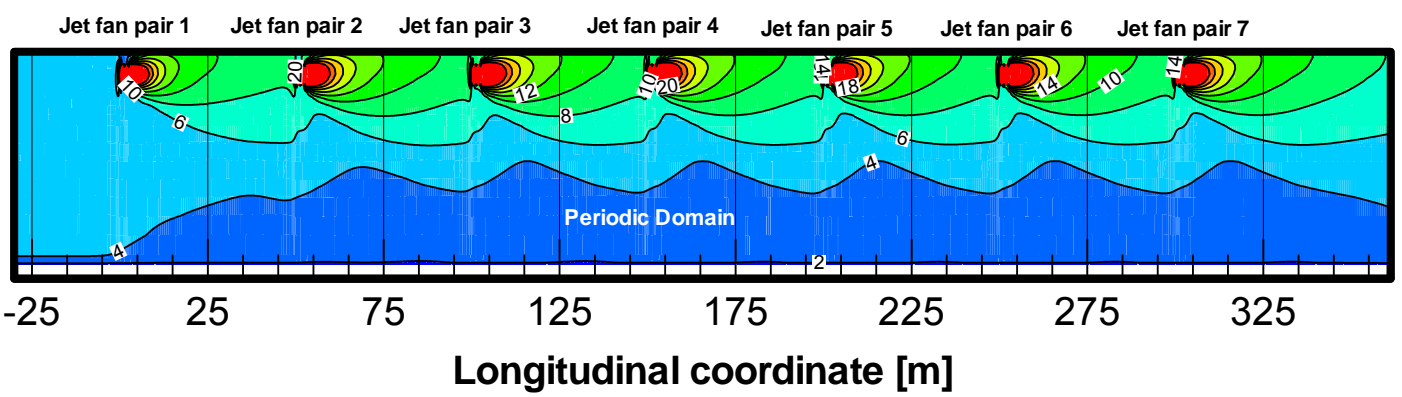

Figure 11 - Typical flow pattern produced by a series of seven operational jet fan pairs. The tunnel height is $6.5 \mathrm{~m}$ (vertical coordinate is scaled up 5 times). Velocity Isocontour from $2 \mathrm{~m} / \mathrm{s}$ to $20 \mathrm{~m} / \mathrm{s}$ in steps of $2 \mathrm{~m} / \mathrm{s}$

the inlet and outlet boundaries have been defined as periodic surfaces. Thus, a jet fan series can be modelled by including a single representative module which operates in a periodic behaviour.

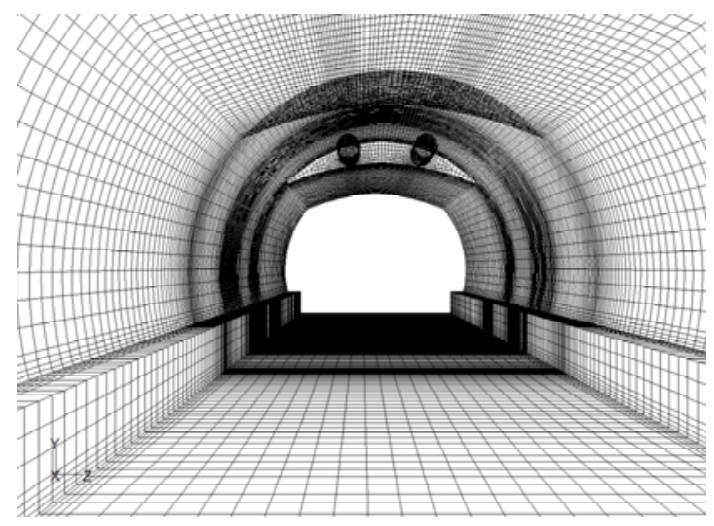

Figure 12 - Example of the mesh in the near field of a jet fan pair and niches

The assumption of periodic flows implies that the velocity components repeat themselves in space. The pressure drop across the modules is periodic. This modelling approach is usually applied for periodic flows where a periodic pressure drop occurs across translationally repeated boundaries [21].

Thus, once the near field has been identified, the CFD mesh has been built following the available tunnel geometric data, in order to represent the jet fans installations and obtain a better estimation of the jet fans thrust. An example of the CFD mesh of the near field is presented in Fig. 12. The niche where the jet fan pair is installed is clearly shown. Several runs of the CFD model of the near field have been performed, varying the pressure difference across the domain boundaries. The results can be presented in terms of bulk flowvelocity and pressure difference across the domain (Fig. 13). The curve obtained describes the capability of each pair of jet fans to produce thrust and its dependence on the bulk

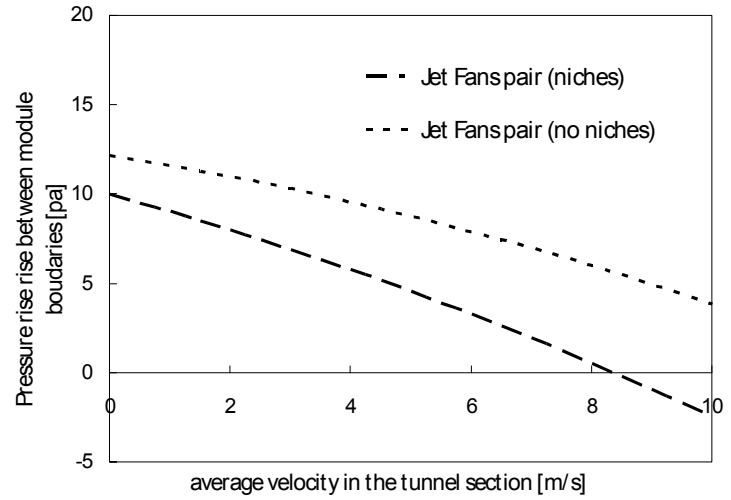

Figure 13 - Characteristic curve of pressure rise vs. flow velocity calculated using CFD of a tunnel module $50 \mathrm{~m}$ in length and containing a jet fan pair, with and without modelling the niches

flow velocity. Another curve has been calculated using a mesh geometry where the jet fans were not installed in niches. The second curve is higher than the first one confirming that the niches significantly influence the pressure rise that the jet fans can produce.

The results of this CFD study for the near field are then coupled to the mono-dimensional model built for the rest of the tunnel.

Specifically, the computed curves are used as the characteristics of any branch of the monodimensional model containing a jet fan pair instead of the classical momentum formulation as described in equation (2), which is not able to properly describe such a complex 3D behaviour. It is worth to highlight that the assumed flow periodicity lasts as long as the supply fans within the tunnel are off. The introduction of fresh air will slightly modify the velocity patterns within the tunnel and the mass flow rate will not be constant along the longitudinal direction. However, these effects are really small as the amount of fresh air introduced in the fan region is negligible compared to the large mass flow rate through the main gallery. Thus, in this case, the computed characteristic curve provides a good approximation which can be successfully applied. 


\subsubsection{Comparison to experimental data}

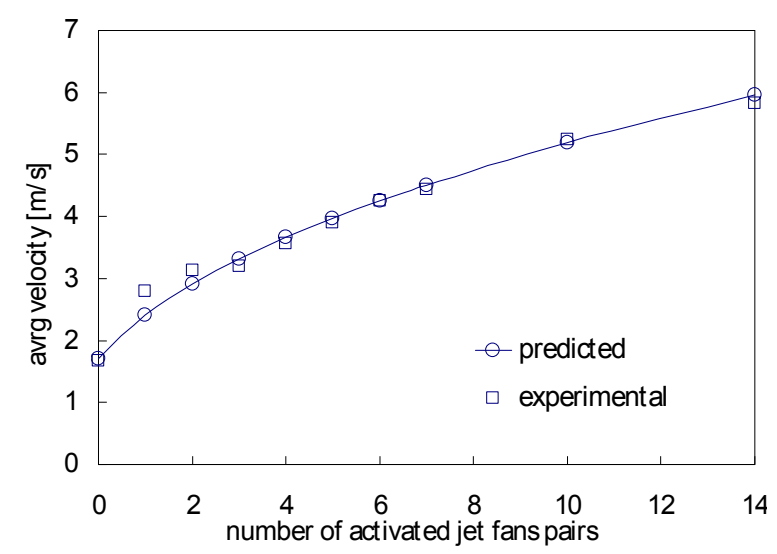

Figure 14 - Comparison of results for the average flow velocities vs. operational jet fan pairs between experimental measurements and CFD predictions

The multi-scale model has been validated using bulk flow data recorded in the central section of the tunnel (Fig. 14). The comparison between the two sets of data is excellent, demonstrating the accurate prediction capabilities of the model.

The simplicity of the model and its robustness allow the simulation of many different ventilation scenarios as well as the effect of different fan combinations, or their interaction with extraction and supply fans; the effect of wind or other different external boundary conditions (difference between static pressures at the adits) can also be taken into account as well as the influence of the vertical shafts and stack effect, dampers and any obstacle within the tunnel. The model can also be set in order to calculate the distribution of pollutants or the influence of the traffic flow on the average air velocity within the tunnel as well as to make real time prediction of the ventilation flows for control purposes. In this work the multi-scale model has been used to assess the performance of the ventilation system of the studied tunnel.

\section{Assessment of the ventilation system of the studied tunnel}

In case of an incident, the emergency ventilation system of the studied tunnel has to push any smoke produced (or gas released) away from the incident region in the same direction as the traffic flow (from south to north) avoiding the smoke spreading against the main air flow. The vehicles downstream the fire zone are assumed to leave the tunnel safely. Most of the studies of tunnel ventilation [3-6] show that air velocities in the region of $2.5 \mathrm{~m} / \mathrm{s}-3 \mathrm{~m} / \mathrm{s}$ are sufficient to avoid back-layering in most fire scenarios. Thus, an adequate ventilation system has to provide air velocities higher than the critical values in the locality of the incident.

Depending on the incident location different ventilation strategies can be adopted. The actual ventilation strategies consider the tunnel domain divided in 4 different Zones (Fig. 4):

- Zone A is located between southern portal and southern shaft. In this scenario the ventilation strategy uses all the jet fan pairs (blowing south to north) and both the extract fans. No supply fans are operational

- Zone B is located between the southern shaft and the tunnel mid point. In this scenario the ventilation strategy uses all the jet fan pairs (blowing south to north) and only the northern extract fan operational. No supply fans are operational

- Zone $\mathrm{C}$ is located between the tunnel mid point and the northern extract shaft. In this scenario the ventilation strategy uses all the jet fan pairs (blowing south to north), the northern extract fan and the southern supply fan

- Zone $\mathrm{D}$ is located between the northern extract shaft and the northern portal. In this scenario the ventilation strategy uses all the jet fan pairs (blowing south to north) and both the supply fans. No extract fans are operational.

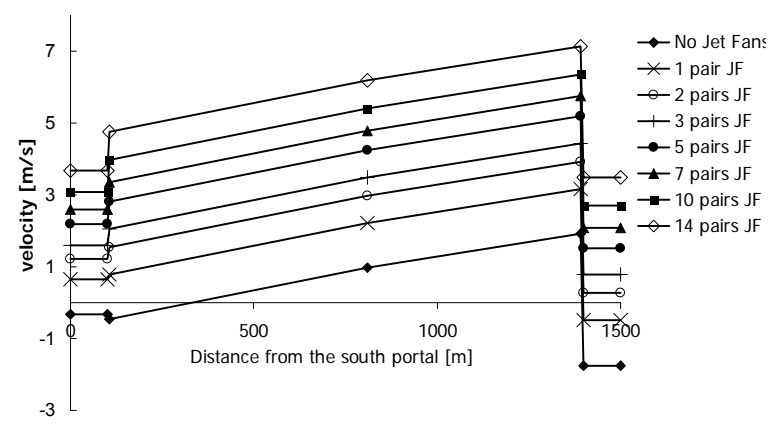

Figure 15 - Predictions of average flow velocity vs. distance for different operational jet fan pairs (ventilation strategies) in Zone $\mathrm{C}$ of the tunnel, (Zone C extends approximately from $700 \mathrm{~m}$ to $1400 \mathrm{~m}$ from the South portal)

Using the model all the ventilation strategies have been analysed and it has been demonstrated that the actual ventilation system, if fully operational, is able to provide sufficient ventilation in all zones. The results of the analysis are shown in Table 1. The model has been also used to asses the redundancy of the system. For each ventilation scenario the minimum number of jet fans required 
Table 1

Summary of the average flow velocities for different ventilation scenarios. The embolded velocities refer to the tunnel zone objective of the respective strategy.

\begin{tabular}{|c|c|c|c|c|c|c|c|c|}
\hline \multirow[t]{2}{*}{ Ventilation strategy } & \multirow[t]{2}{*}{ All jet fans } & \multirow[t]{2}{*}{ South extract fan } & \multirow[t]{2}{*}{ North Extract fan } & \multirow[t]{2}{*}{ South supply fan } & \multirow[t]{2}{*}{ North supply fan } & \multicolumn{3}{|c|}{ Ventilation velocities $[\mathrm{m} / \mathrm{s}]$} \\
\hline & & & & & & Zone $\mathrm{A}$ & Zone $\mathrm{B} / \mathrm{C}$ & Zone D \\
\hline Zone A & $\mathrm{X}$ & $\bar{x}$ & $\bar{x}$ & - & - & 9 & 5.1 & 1.2 \\
\hline Zone B & $\mathrm{x}$ & - & $\mathrm{x}$ & - & - & 4.5 & 5.9 & 2 \\
\hline Zone C & $\mathrm{x}$ & - & - & $\mathrm{x}$ & - & 3.5 & 5.9 & 3.3 \\
\hline Zone D & $\mathrm{x}$ & - & - & $\mathrm{x}$ & $\mathrm{x}$ & 2.2 & 5.4 & 5.1 \\
\hline JF only & $\mathrm{x}$ & - & - & - & - & 4.2 & 5.6 & 3.6 \\
\hline
\end{tabular}

to reach critical velocity has been calculated. The computed data are significant because the effect of jet fans failures during an emergency scenario can easily be assessed. The redundancy has been calculated for all the 4 ventilation scenarios but the results presented in Fig. 15 are for an incident in zone C. Assuming $3 \mathrm{~m} / \mathrm{s}$ as minimum velocity to avoid back-layering, it appears that at least two pairs of jet fans are required to maintain sufficient ventilation flow in the tunnel. It must be stressed that all the calculations presented were performed for the case of cold flow in the tunnel, which is completely free from traffic or other obstructions. In a real emergency scenario the tunnel would, most likely, be full of vehicles producing further losses and resistance to the flow. Furthermore, the additional losses induced by a fire must be considered. They have been studied many decades ago [27], when experiments have confirmed the fire 'throttling' effect. This effect will obviously increase with the fire size and would tend to reduce the flow in the tunnel.

Thus, the ability to provide critical velocity in cold flow conditions does not necessarily guarantee that such flow conditions can be assured under emergency conditions. Studies performed using a multi-scale modelling approach [28] and including the effect of the fire have confirmed that cold flow calculations can significantly under-predict the minimum ventilation requirement to control the smoke spread. However such considerations are out with the scope of this work.

\section{Conclusions}

Two different multi-scale techniques to model ventilation flows within road tunnels have been discussed and validated using experimental data recorded in a real tunnel.

The first multi-scale model uses direct coupling between a monodimensional model and a CFD model and it has been adopted to simulate the velocity field generated by an operating pair of jet fans in a real tunnel. The solving procedure, the effect of the interfaces location as well as the effect of the mesh size have been discussed. The predicted velocity values have been compared with experimental measurements taken within the tunnel. The paper demonstrates that the multiscale approach can lead to very accurate results with a significant reduction of the computational requirements.

The second multi-scale model uses indirect coupling between a mono-dimensional model and a CFD model. The multi-scale model has been used first to reconstruct the behaviour of a pair of jet fans in order to obtain a better estimation of the thrust and its dependence on the bulk flow velocity. The model shows that the niches where the fans are installed produce a significant reduction of the ventilation system performance due to a decrease of the thrust of the jet fans. The results of the model have been validated under different ventilation scenarios using bulk flow data recorded within the tunnel.

The models have been demonstrated to be a valid tool for the simulation of the complex behaviour of the tunnel ventilation systems. They can be successfully adopted to design ventilation systems and to assess their redundancy and their performance under different operative conditions. An example of performance assessment has been performed in the case of the studied tunnel. The analysis demonstrates the capability of the actual ventilation system to provide adequate levels of flow for all the incident ventilation strategies. The model has also demonstrated that, for a given ventilation scenario, even if there are some jet fan failures, the tunnel ventilation system will still be able to provide adequate air flow for the incident ventilation strategy.

\section{Acknowledgements}

The authors would like to thank Hubert Biteau, Pedro Reszka, Thomas Steinhaus, Nicolas Bal, Cristian Maluk, Kate Anderson andAdam Cowlard for several nights spent in a cold tunnel and their precious help with the on-site measurements.

\section{Appendix A. - Numerical features of the mono- dimensional model}

Tunnel ventilation systems very often can present complex interconnections and many internal loops. In order to easily describe the connection pattern between branches and nodes the model uses a matrix formulation based on a connection pattern matrix $A$ which summarizes the layout of 
the system. The whole set of mass conservation equations can be described as follows

$$
[A]\{G\}+\left\{G_{e x t}\right\}=\{0\}
$$

where the terms in curly brackets respectively represent a vector containing the branches flows and a vector made by the flows exchanged with the ambient in each node. The same matrix formulation can be used to represent the set of momentum equations. After linearizing all the terms contained in equation (2), and some rearrangements the complete set of momentum equations can be presented as

$$
[A]^{T}\{p\}=[Y]\{G\}+\{M\}
$$

where the pressure difference term on the $L H T$ is function of the mass flow rate in each branch plus a constant term $M$. Rearranging equation (7) the mass flow rate in each branch can be then expressed as

$$
\{G\}=[Y]^{-1}[A]^{T}\{p\}+[Y]^{-1}\{M\}
$$

The iterative solution strategy known as the SIMPLE algorithm [22] is adopted to solve the system. The method, based on a 'guess and correct' procedure [24] and its rearrangement for a monodimensional network model is presented here.

The iterative procedure starts with a guess of the pressure field $p^{*}$ which allows the calculation of the guessed flow field $G^{*}$.

$$
\left\{G^{*}\right\}=[Y]^{-1}[A]^{T}\left\{p^{*}\right\}+[Y]^{-1}\{M\}
$$

Then, the pressure correction $p_{0}$ and the flow correction $G_{0}$ are defined as difference between correct and guessed values. After some rearrangements a relation between mass flow rate and pressure correction can be obtained

$$
\{G\}=\{G *\}+[Y]^{-1}[A]^{T}\left\{p^{\prime}\right\}
$$

By substituting equation (10) in the continuity equation (6), after some rearrangements, the pressure correction equations can be written as

$$
[A][Y]^{-1}[A]^{T}\left\{p^{\prime}\right\}=-[A]\left\{G^{*}\right\}-\left\{G_{e x t}\right\}
$$

At each iteration step, equation (11) is used to calculate the pressure correction to update pressure and flow fields. Obviously, in the nodes where the pressure values are known (i.e. the boundary nodes), the pressure correction is set to zero.
The developed numerical algorithm requires under relaxation in order to reach convergence. In particular the new update values for pressure and velocities are calculated by means of relaxation factors that can provide more stable computations.

The main steps of the solution procedure of the mono-dimensional model are resumed as follows:

1. guess a pressure field $p^{*}$

2. solve the momentum equations to obtain $G^{*}$ (equations (9))

3. solve the pressure correction equations to calculate $p_{0}$

4. (equation (11))

5. update pressures and velocities

6. solve energy equations and update temperatures and densities

7. iterate from step 2 to step 5 until convergence is reached.

\section{References}

1. PIARC Committee on road tunnels. Fire and smoke control in road tunnels;2004.

2. Li JSM, Chow WK. Numerical studies on performance evaluation of tunnel ventilation safety systems. Tunnelling and Underground Space Technology 2003; 18(5):435-52.

3. Thomas $\mathrm{PH}$. The movement of buoyant fluid against a stream and venting of underground fires. Fire Research note 1958:351.

4. Kunsch JP. Simple model for control of fire gases in a ventilated tunnel. Fire Safety Journal 2002;37(1):67-81.

5. Oka Y, Atkinson GT. Control of smoke flow in tunnel fires. Fire Safety Journal 1995;25(4):305-22.

6. Vaquelin $\mathrm{O}, \mathrm{Wu} \mathrm{Y}$. Influence of tunnel width on longitudinal smoke control. Fire Safety Journal 2006;41(6):420-6.

7. Massachusetts Highway Department. Memorial tunnel fire ventilation test program comprehensive test report; 1995.

8. EUREKA 499 Report. Fire in transport tunnels, report on full scale tests. Duesseldorf: Studiengesellschaft Stahlanwendung e.V.; 1996.

9. Ferro V, Borchiellini R, Giaretto V. Description and application of tunnel simulation model. Aerodynamics and ventilation of vehicle tunnels. London: Elsevier Science Publishers Ltd; 1991. p. 487-12.

10. Jacques E. Numerical simulation of complex road tunnels. Aerodynamics and ventilation of vehicle tunnels. London: Elsevier Science Publishers Ltd; 1991. p. 467-86. 
11. Riess I, Bettelini M, Brandt R. SPRINT - a design tool for fire ventilation. In: Tenth international symposium on aerodynamics and ventilation of vehicle tunnels. Boston, USA; 2000.

12. Cheng LH, Ueng TH, Liu CW. Simulation of ventilation and fire in the underground facilities. Fire Safety Journal 2001;36(6):597-619.

13. Jang $H$, Chen $F$. On the determination of the aerodynamic coefficients of highway tunnels. Journal of Wind Engineering and Industrial Aerodynamics 2002;89(8):86996.

14. Armstrong J. The ventilation of vehicle tunnels by jet fans - the axisymmetric case. In: Proceedings of the seminar on installation effects in fan systems. London: Mechanical Engineering Press; 1993.

15. Tabarra M. Optimizing jet fan performance in longitudinally ventilated rectangular tunnels. Separated and complex flows. ASME FED 1995;217:3542.

16. Karki KC, Patankar SV. CFD model for jet fan ventilation systems. In: Tenth international symposium on aerodynamics and ventilation of vehicle tunnels. Boston, USA; 2000.

17. Galdo Vega M, Arguelles Diaz KM, Fernandez Oro JM, Ballesteros Tajadura R, Santolaria Morros C. Numerical 3D simulation of a longitudinal ventilation system: memorial tunnel case. Tunnelling and Underground Space Technology 2008;23(5):539-51.

18. Formaggia L, Gerbeau JF, Nobile F, Quarteroni A. On the coupling of 3D and 1D Navier-Stokes equations for flow problems in compliant vessels. Computer Methods in Applied Mechanics and Engineering 2001;191(6-7):561-82.

19. Formaggia L, Nobile $F$, Veneziani A. Multiscale modelling of the circulatory system: a preliminary analysis. Computing and Visualization in Science 1999;2:75-83.

20. Fox RW, McDonald AT. Introduction to fluid mechanics. 4th ed. Singapore: John Wiley and Sons; 1995.

21. Fluent Inc. FLUENT 6.2 user's guide; 2005.

22. Patankar SV, Spalding DB. A calculation procedure for heat, mass and momentum transfer in three-dimensional parabolic flows. International Journal of Heat and Mass Transfer 1972;15:1787-806.

23. Launder BE, Spalding DB. The numerical computation of turbulent flows. Computer Methods in Applied Mechanics and Engineering 1974;3(2): 269-89.
24. Versteeg HK, Malalasekera W. An introduction to computational fluid dynamics, the finite volume method. 2nd ed. Glasgow: Pearson Prentice Hall; 2007.

25. Lacasse D, Turgeon E, Pelletier D. On the judicious use of the $k-\varepsilon$ model, wall function and adaptivity. International Journal of Thermal Sciences 2004;43(10):925-38.

26. Schlichting $\mathrm{H}$. Boundary layer theory. 7th ed. New York: McGraw-hill; 1979.

27. Lee CK, Chaiken RF, Singer JM. Interaction between duct fires and ventilation flow: an experimental study. Combustion Science \& Technology 1979; $20(1-2): 59-72$.

28. Colella F, Borchiellini $R$, Carvel $R$, Rein G, Torero JL, Verda V. Two-scale modelling approach for simulating flows in tunnel fires. International congress in smoke control in buildings and tunnels. Santander; Oct. 2008. Copy available at http://www.era.lib.ed.ac.uk/handle/1842. 